\title{
Ameloblastoma of the mandible: A case series
}

\author{
Mosmi Lomi ${ }^{1, *}$, Sankari Radhakrishnan², VK Jimsha ${ }^{3}$, V. Ramesh ${ }^{4}$, M. Jonathan Daniel ${ }^{5}$ \\ ${ }^{1,2}$ Post Graduate Resident, ${ }^{3}$ Assistant Professor, ${ }^{4}$ Dean, ${ }^{\mathbf{5}}$ Associate Dean, ${ }^{\mathbf{1}, 3,5}$ Dept. of Oral Medicine \& Radiology, ${ }^{2,4}$ Dept. of Oral \\ Pathology \& Microbiology, Mahatma Gandhi Postgraduate Institute of Dental Sciences, Pondicherry, Puducherry, India
}

\section{*Corresponding Author: Mosmi Lomi}

Email: nanulomi@gmail.com

\begin{abstract}
Ameloblastoma is a benign odontogenic neoplasm which exhibits a locally aggressive behaviour. It commonly affects the mandible and seen in third to fifth decades of life. In most cases, an ameloblastoma is usually asymptomatic and may be detected during the course of routine radiographic examination. Radiographically, it may have a unilocular or multilocular appearance, which is usually well defined and have corticated margin in the mandible whereas ill-defined in case of maxilla. In this case series, we report 3 cases of ameloblastoma of the mandible.
\end{abstract}

Keywords: Ameloblastoma, mandible, Multilocular radiolucency, Bicortical expansion.

\section{Introduction}

Ameloblastoma is a locally destructive, benign neoplasm arising from odontogenic epithelial origin ${ }^{1}$ which derived its name from the old French word "amel" means enamel and the Greek word "blastos" means germ or bud. This neoplasm was first described by Cusack in $1827^{2}$ and was named as an "adamantinoma" by Malassez in 1885 , which is currently used to name a rare form of bone cancer described by Fisher (1913). Later in 1930, Ivey and Churchill coined the term "ameloblastoma" which is currently preferred term. The World Health Organization in 1991 defined ameloblastoma as a benign but locally aggressive tumor with a high tendency to recur, consisting of proliferating odontogenic epithelium lying in a fibrous stroma. ${ }^{3}$ In this case series, we report three male patients who were diagnosed with ameloblastoma of the mandible at our institute.

\section{Case Report \\ Case 1}

Eighteen year old male patient reported in our department with swelling in left lower jaw since past 6 months. Swelling was gradual in onset, slowly progressed to attain the present size and was not associated with pain. Patient was known case of seizure disorder and was under appropriate medication since past 7 months. On extraoral examination, facial asymmetry was seen. A diffused swelling was present on the left lower third of the face and the swelling was extending anteriorly to the left corner of the mouth and posteriorly to the left pretragus region, superiorly $2 \mathrm{~cm}$ below the zygoma and inferiorly to the inferior border of the mandible. Skin over the swelling appeared normal without any secondary changes. On palpation, swelling was non tender, firm to hard in consistency. Intraorally, a diffused swelling was present in the buccal aspect of teeth 35,36 , edentulous area of teeth 37, 38 and extending upto retromolar area. Overlying mucosa showed an indentation of the opposite teeth (Fig. 1a). On palpation, swelling was non tender, firm to hard in consistency with few soft spots on the buccal aspects and showed bucco-lingual expansion. Clinically teeth 37,38 were missing with no past history of extraction. On the basis of history and clinical findings, a provisional diagnosis of dentigerous cyst in relation to teeth 37,38 was given.

Vitality testing showed no response in relation to tooth 36 and immediate response in relation to tooth 35 . Lesional aspiration yielded a high viscous mucous fluid mixed with blood aspirate.

Occlusal radiograph showed well defined multilocular radiolucency with buccal and lingual cortical expansion in relation to teeth $36,37,38$. Panoramic radiograph revealed well defined multilocular radiolucency with coarse and curved internal septation, extending from distal aspect of teeth 34 to pericoronal aspect of 38 , displaced teeth 37 and 38. The inferior alveolar nerve canal was displaced downward and inferior border of mandible in relation to teeth 37, 38 was ballooning out and intact (Fig. 1b). CBCT revealed hypodense area in relation to left side of the body \& angle of the mandible with bicortical expansion \& thinning of buccal and lingual cortex without perforation (Fig. 2a).

Histopathological examination showed dense collagenous fibrous connective tissue with a well defined follicle noticed in the form of island which has peripherally placed ameloblast like cells with reverse polarity and centrally placed stellate reticulum exhibiting microcystic changes suggestive of follicular ameloblastoma (Fig. 2b). 


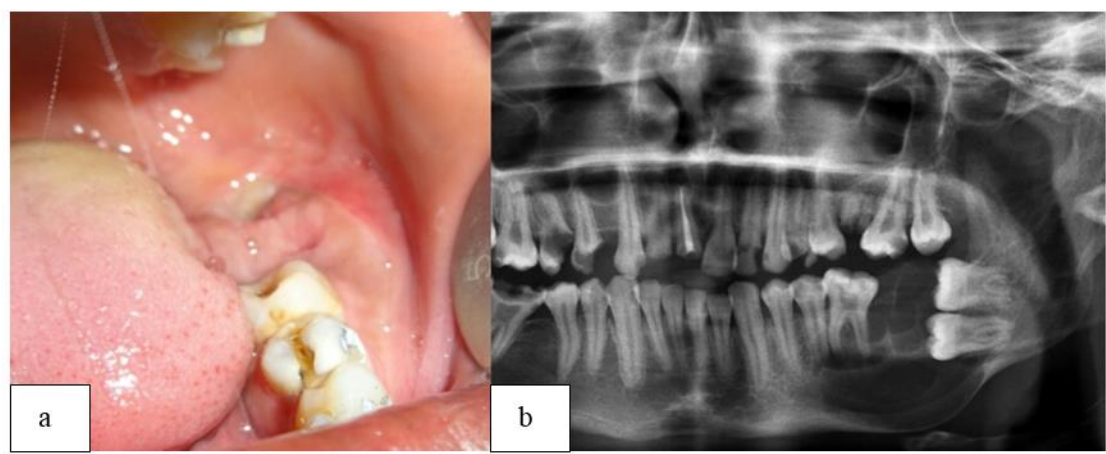

Fig. 1: a): Overlying mucosa showing an indentation of the opposite teeth; b): Panoramic radiograph showing multilocular radiolucency with coarse \& curve internal septation, displaced teeth 37,38 , downward displacement of inferior alveolar nerve canal

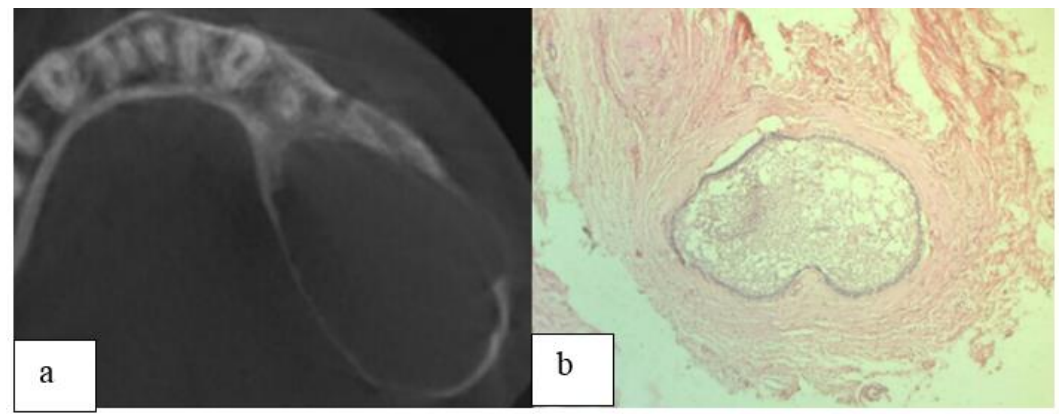

Fig. 2: a) Axial section of CBCT showing hypodense area with bicortical bone expansion without perforation. Histological picture showing collagenous fibrous connective tissue with follicle in the form of island which has peripherally placed ameloblast like cells with reverse polarity and centrally placed stellate reticulum exhibiting microcystic changes

\section{Case 2}

Thirty three year old male patient presented in our department with chief complaint of swelling in lower jaw since past 9 months. Initially swelling was small in size, then it gradually increased over the past several months to attain its present size. Swelling was not associated with pain, numbness and paraesthesia. Patient gave history of trauma before 20 years on the chin. On extraoral examination, there was a diffused swelling on left side of lower third of the face of size measuring $4 \times 3 \mathrm{~cm}$ with obliteration of mentolabial sulcus which extended anteroposteriorly from symphysis to left corner of the mouth, superiorly to vermillion border of lower lip, inferiorly to the inferior border of the mandible. Surface of the swelling was smooth with no visible pulsation and secondary changes. On palpation swelling was non tender, firm to hard in consistency with localised elevated temperature. On intraoral examination, swelling was present in the lower jaw in relation to teeth 43 to 35 of size measuring $5 \times 2 \mathrm{~cm}$ which extended anteroposteriorly from mesial aspects of 43 to distal aspect of 35, superoinferiorly from attached gingiva to vestibule (Fig. 3a). Lingually swelling extent from distal aspect of teeth 31 to mesial aspect of 34, superoinferiorly from below marginal gingiva to floor of the mouth. Surface of the swelling was smooth. Buccally, swelling was non tender, soft to firm in consistency with soft spot in relation to teeth 34,35 on palpation, lingually it was non tender, hard in consistency. Gingival hypertrophy of size $0.5 \mathrm{~cm}$ was present in relation to teeth 31,32 on the buccal aspect which was erythematous, pedunculated, irregular in shape (Fig. 3a). On palpation it was non tender, firm in consistency. The teeth $31,32,33,41$ and 42 were mobile with discoloration in relation to teeth 31,41 . The teeth $31,32,41$ were displaced with tenderness on percussion. On the basis of history and clinical findings, a provisional diagnosis of radicular cyst in relation to $31,32,41$ was given.

Vitality test showed no response in relation to teeth 31 , $32,41,42$. Lesion aspiration yielded a blood mixed thin fluid aspirate.

Occlusal radiograph revealed well defined multilocular radiolucency with granular septae, extending from distal aspect of teeth 42 to mesial aspect of 37 . Buccal cortical expansion with thinned border and displacement of 32 mesially and 33 distally were seen. Panoramic radiograph revealed well defined unilocular radiolucency extending from mesial aspect of teeth 43 to distal aspect of 35 . The radiolucency extended inferiorly to the lower border of the mandible. Displacement of 32 mesially, 33 distally with resorption of root in relation to teeth $31,32,33,34,35,41$, 42 and loss of lamina dura in relation to teeth $31,32,33,34$, 35, 41, 42,43 were seen (Fig. 3b). CBCT revealed hypodense area in the lower jaw in anterior region crossing the midline with bicortical expansion and thinning of buccal cortex without perforation (Fig. 4a). 
Histopathological examination showed network of odontogenic epithelium connected to each other with prominent peripheral columnar cells with some cells exhibiting reverse polarity suggestive of plexiform type of ameloblastoma (Fig. 4b).

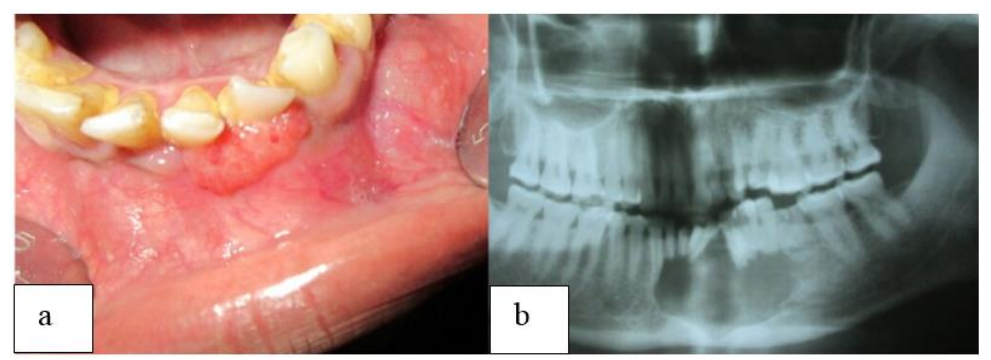

Fig. 3 a): Picture showing swelling in the lower jaw extending from teeth 43 to 35 ; b): Panoramic radiograph showing unilocular radiolucency, displacement of teeth 32, 33, root resorption in relation to teeth 35-42

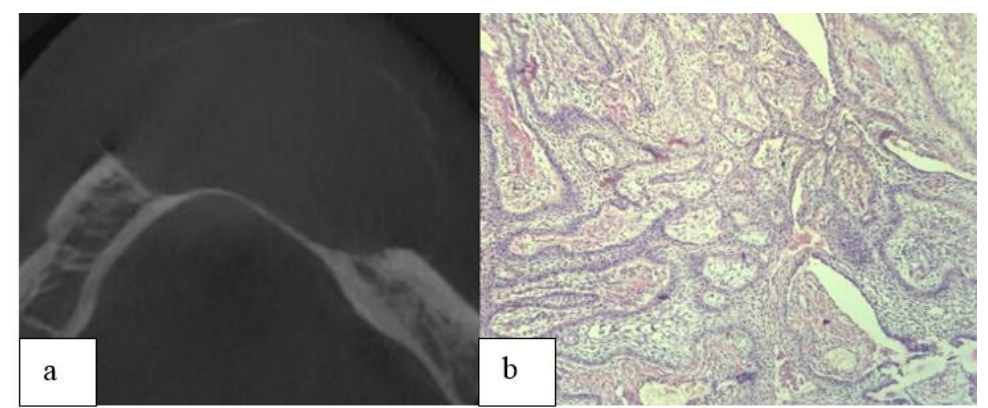

Fig. 4 a): Axial section of CBCT showing hypodense area with bicortical expansion and thinning of buccal cortex; b): Histopathological picture showing network of odontogenic epithelium connected to each other with peripheral columnar cells with some cells exhibiting reverse polarity

\section{Case 3}

Thirty six year old male patient presented with chief complaint of swelling in left side of the lower jaw since past 5 years. Swelling was gradual in onset, slowly progressed to present day size. Swelling was associated with pain since past one and half month. Pain was mild, intermittent which aggravated during chewing food. Past dental history revealed extraction of tooth 36 due to pain and decay before 7 years. There was no evidence of swelling on extraoral examination. Intraorally, a single well defined swelling of size $2 \times 1 \mathrm{~cm}$ was present in left side of the lower jaw extending from teeth 35 to 37 region with obliteration of buccal vestibule (Fig. 5a). Surface of an overlying mucosa was smooth \& red in colour. On palpation swelling was tender, soft to firm in consistency, compressible. Clinically, tooth 36 was missing with history of extraction, teeth $37 \&$ 38 were decayed with no tenderness on percussion. On the basis of history and clinical examination, a provisional diagnosis of infected residual cyst in relation to tooth 36 was given.

Vitality test showed immediate response in all teeth except in 37. Lesion aspiration yielded a blood mixed thick viscous fluid aspirate.

Occlusal radiograph showed well defined multilocular radiolucency extending from distal aspect of teeth 35 to mesial aspect of 37 with buccal cortical expansion and destruction. Panoramic radiograph revealed well defined multilocular radiolucency extending from distal aspect of teeth 35 to mesial aspects of 37 . The internal structure of the multilocularity revealed honeycomb pattern with septation (Fig. 5b). CBCT revealed hypodense area in relation to left side of the body of the mandible with bicortical expansion and destruction of buccal cortex (Fig. 6a).

Histopathological examination showed odontogenic epithelium in the form of islands and network in follicular background. Individual islands exhibited classical peripheral ameloblast like cells and central stellate reticulum like cells suggestive of follicular ameloblastoma (Fig. 6b). 


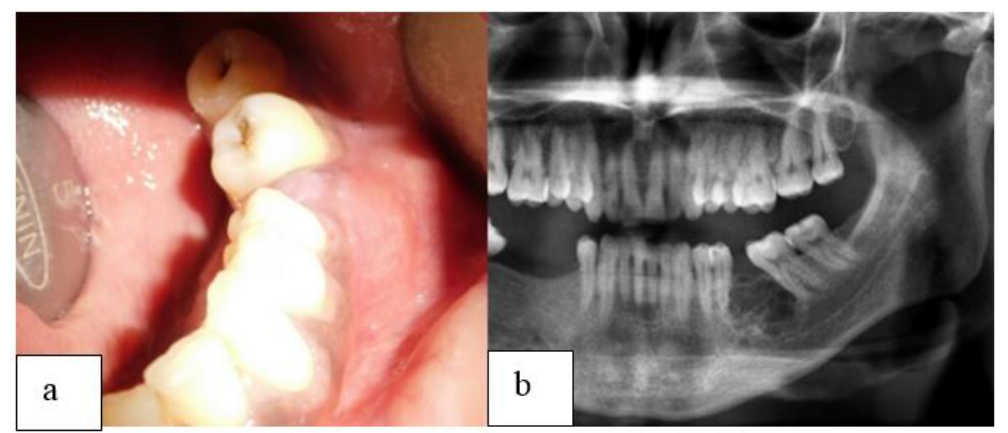

Fig. 5 a): Picture showing swelling in left side of lower jaw with obliteration of buccal vestibule; b) Panoramic radiograph showing multilocular radiolucency with honeycomb pattern internal structure

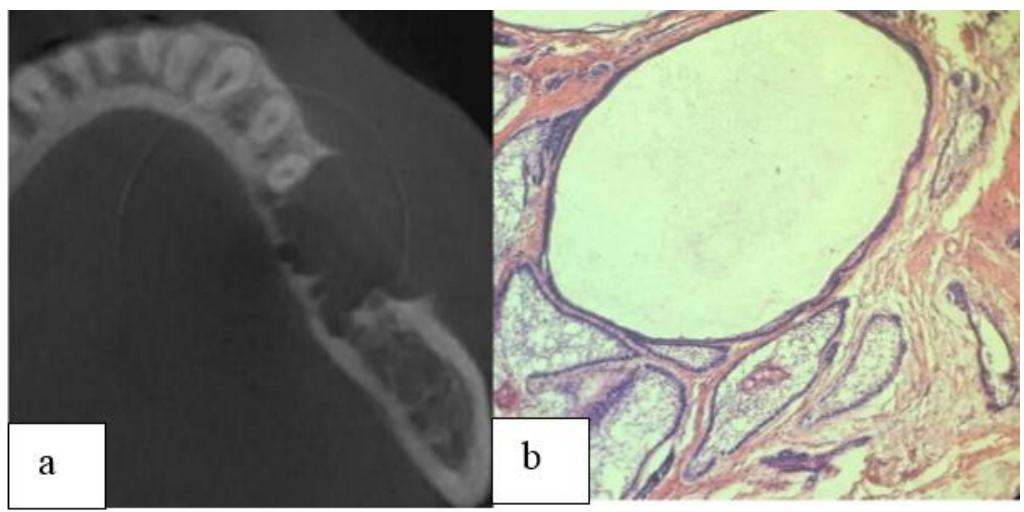

Fig. 6 a): Axial section of CBCT showing hypodense area in the body with bicortical expansion and destruction of buccal cortex; b): Histopathological picture showing odontogenic epithelium in the form of islands and network in follicular background. Individual islands exhibited classical peripheral ameloblast like cells and central stellate reticulum like cells

\section{Discussion}

Ameloblastomas are an enigmatic group of oral tumors that often invade locally and occasionally metastasize but usually has a benign growth pattern. ${ }^{4}$ Based on an analysis of the odontogenic epithelium involvement in the origin of the tumour, Ivy \& Churchill in 1960 proposed the term "ameloblastoma". According to the literature, potential sources of this specialized epithelium may also include from the following: embryonic odontogenic rests (rests of Malassez and rests of Serres), the epithelial lining of odontogenic cysts (especially the dentigerous cyst) and basal cells of the oral mucosa. However, the trigger for neoplastic transformation of these epithelial structures remains unknown. ${ }^{5}$ Ameloblastoma reported to constitutes about $14 \%$ of all jaw tumors and cysts. The global incidence is 0.5 cases per million persons per year. ${ }^{6}$ According to WHO 2017 classification, ameloblastoma are divided into four categories: a) conventional b) extraosseous/peripheral type c) unicystic and d) metastasizing ameloblastoma. ${ }^{7}$

It is commonly seen in the third to fifth decades of life, however it has been reported to be found in age ranging from 10-90 years. ${ }^{4}$ In this series, ameloblastoma was observed to occur in 18-36 years with mean age of 29 years. This finding of our series is not consistent with the studies conducted by Rajeshwar Chawla et al, Chandraman More et al, Soujanya Reddy et al, who found mean age of 32.5 years, 43.5 years, 45 years, respectively. Regarding gender predilection, some authors state a male predilection while others describe it as being more common in females. ${ }^{8}$ In our series, all the 3 cases were seen in male. Above $80 \%$ of ameloblastoma occur in the mandible, of which $70 \%$ arises in the molar-ramus region. It rarely affects the maxilla and the soft tissue (peripheral ameloblastoma). ${ }^{4}$ All the three cases in our series were seen in the mandible, case 1 occurred in premolar-angle region, case 3 occurred in premolar-molar region while case 2 occurred in the mandible involving anterior-left premolar region. The reason why mandible is the favored site for ameloblastoma is still unknown.

Ameloblastoma may be relatively asymptomatic, and when symptoms are present, patient usually complain of a slow growing, painless swelling ${ }^{9}$ as in our case 1 and case 2 . Pain, paraesthesia, loose teeth or malocculusion may present but are less common and facial deformity, if present, may range from very mild to severe in delayed presentations. ${ }^{9}$ In case 2 and case 3, loose teeth and mild pain were present respectively. According to White and Pharoah and Kim and Jang, around $10-15 \%$ may be associated with a unerupted tooth, often a third molar of the mandible. ${ }^{8}$ Here Case 1 was associated with impacted second and third molar.

In most cases, ameloblastoma present a characteristic, though not diagnostic, radiographic appearance. Radiographically, the lesion in the mandible have usually well defined, frequently corticated and occasionally scalloped margins. In contrast to the mandible, the margins 
in the maxilla are severely ill-defined as the lesion tends to grow along the bone rather than expanding it. The internal structure of the lesion varies from complete radiolucency to mixed, with the presence of bony septa that create internal compartments. The septae originate from the normal bone that has been trapped within lesion, and are usually coarse and curved. With growth or expansion of the tumour, there may be coalescence and fusion of the compartments, and as a results, the lesion may transform from multilocular to a monolocular cystic space. Tumours with large and few compartments may resemble a multilocular epithelium lined cyst. ${ }^{4}$

Tooth displacement, inferior alveolar canal displacement or destruction and displacement of the sinus membrane are the common findings seen in ameloblastoma. $^{4}$ In this series, case 1 and case 2 radiographically showed inferior alveolar nerve canal displacement \& teeth displacement respectively. Melvor stated four radiographic features which suggest that a radiolucent lesion is an ameloblastoma and these include expansion of cortical plate, presence of corticated scalloped margins, multilocular appearance of lesion and the resorption of adjacent roots of teeth. ${ }^{1}$ Out of three cases in our series, all three cases showed bicortical expansion, one case showed buccal cortical perforation, one case showed resorption of adjacent roots of teeth whereas all the three cases showed well defined multilocular appearance but with non scalloped borders.

Ameloblastoma frequently recurs after treatment. Treatment ranges from conservative surgery to radical procedures with rate of recurrence of $17.7 \%$ for en bloc resection whereas $34.7 \%$ for conservative therapy. Factor such as size of the tumor, presence of ruptured basal cortical bone and histological pattern seems to be an important for the management. ${ }^{10}$

\section{Conclusion}

Ameloblastoma is a benign neoplasm of the mandible and maxilla with a well-documented propensity for locoregional invasion and risk of recurrence. It is the second most common benign odontogenic neoplasm with the primary site being the mandible. Well defined multilocular radiolucency with non scalloped border and bicortical expansion were the common radiological features seen in our series. Very often the diagnosis of ameloblastoma is made on the basis of radiographic features, however one should never rely on it alone. Combination of radiographic evaluation and histopathological examination should be done to provide a correct diagnosis.

\section{Conflict of Interest: Nil.}

\section{References}

1. S. Jayachandran, Khushboo Singh. "Imaging analysis of ameloblastoma of mandible- 5 cases". J Anal Oncol. 2012 Vol. 1, No.2 164-168

2. Andrew C. McClary, Robert B. West, Ashley C. McClary. "Ameloblastoma: A clinical review and trends in management". Eur Arch Otorhinolaryngol. 2016;273(7):16491661

3. Masthan K, Anitha N, Krupaa J et al. “Ameloblastoma”. J Pharm Bioall Sci. 2015;7 S167-170

4. Chandramani More, Mansi Tailor, Hetul J Patel. "Radiographic analysis of ameloblastoma: A retrospective study". Indian J Dent Res. 2012;23(5).

5. N.P. Prabhu, Vijay Ebenezer, R. Balakrishnan. "Ameloblastoma: Report of two cases and a brief literature review”. Biomedical \& Pharmacol J. 2014;7(1):225-230.

6. OA Effiom, OM Ogundana, AO Akinshipo. "Ameloblastoma: current etiopathological concepts and management". Oral Diseases. 2018;24:307-316.

7. Wright JM, Soluk Tekkesin M. "Odontogenic tumors. Where are we in 2017?". J Istanb Univ Fac Dent. 2017;51(3 Suppl 1):S10-S30.

8. Nigel R. Figueiredo, Ajit D. Dinkar, Manoj Meena. "Ameloblastoma: A clinicoradiographic and histopathologic correlation of 11 cases seen in Goa during 2008-2012". Contemp Clin Dent. 2014;5(2)

9. Mir Ruquaya, Vikram Pratap Singh. “Ameloblastoma-A locally destructive and invasive tumour- Review of literature". Int J Otolaryngol Head Neck Surg. 2014;3:216-222.

10. Soujanya Reddy, Smrithi Devi Veera, Gouramma padanad. "Clinicopathological and imaging variants of Ameloblastoma Case series”. Int J Appl Dent Sci. 2015;1(4):145-148.

How to cite this article: Lomi M, Radhakrishnan S, Jimsha V.K, Ramesh V, Daniel M. J. Ameloblastoma of the mandible: A case series. Int $\mathrm{J}$ Oral Health Dent. 2018;4(4):242-246. 